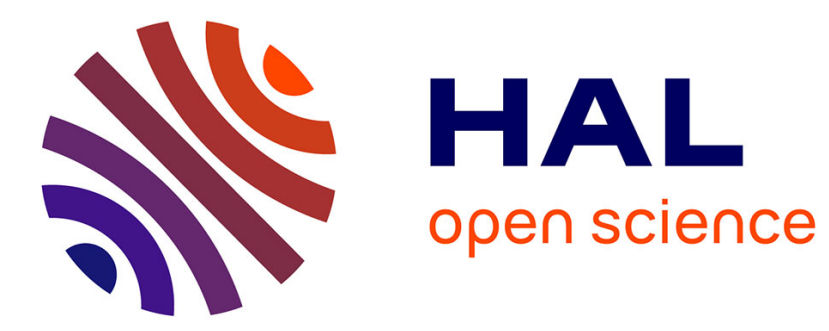

\title{
Model-Driven Alignment: An Empirical Study
}

Constantinos Giannoulis, Jelena Zdravkovic

\section{To cite this version:}

Constantinos Giannoulis, Jelena Zdravkovic. Model-Driven Alignment: An Empirical Study. 7th IFIP Working Conference on The Practice of Enterprise Modeling (PoEM), Nov 2014, Manchester, United Kingdom. pp.271-281, 10.1007/978-3-662-45501-2_20 . hal-01282006

\section{HAL Id: hal-01282006 https://hal.inria.fr/hal-01282006}

Submitted on 3 Mar 2016

HAL is a multi-disciplinary open access archive for the deposit and dissemination of scientific research documents, whether they are published or not. The documents may come from teaching and research institutions in France or abroad, or from public or private research centers.
L'archive ouverte pluridisciplinaire HAL, est destinée au dépôt et à la diffusion de documents scientifiques de niveau recherche, publiés ou non, émanant des établissements d'enseignement et de recherche français ou étrangers, des laboratoires publics ou privés. 


\title{
Model-driven Alignment: An Empirical Study
}

\author{
Constantinos Giannoulis, Jelena Zdravkovic \\ Department of Computer \& Systems Sciences, \\ Stockholm University \\ Forum-16440, Kista, Sweden \\ \{constantinos, jelenaz\}@dsv.su.se
}

\begin{abstract}
Current advancements in the business arena necessitate more than ever before the alignment of The Business and IT in organizations, which has been acknowledged as a complex issue to address. Our research is aimed at systematically addressing the linkage between business strategy and information systems (IS). We propose a model-driven approach for alignment, by leveraging the influence of established business strategy formulations from Strategic Management, and model-driven principles used within IS. The objective of this paper is to present the results of an empirical investigation carried out in Sweden on the linkage seeking to obtain insights from practitioners about the relevance of the problem, as well as of our model-driven proposal to address it.
\end{abstract}

Keywords: Business Strategy, Alignment, IS, Models, UBSMM

\section{Introduction}

Information technology (IT) is a fundamental factor for business strategy enactment [1], because it pervades all sectors of organizations regardless of the organization's business, and hence influences the strategy itself. IT comprises the essential information needed to build executable information systems (IS) to support and facilitate business operations for delivering offerings to customers.

Business strategy should be understood and communicated in an organization to define the means required for its successful execution, also making clear for IT what business stakeholders need. This is expressed through business strategy formulations such as the Value Chain [2], Strategy Maps and Balanced Scorecards (SMBSC) [3]). Organizations aligning their business strategy to IT tend to outperform those that do not [4] and increase their performance and profits [5]. While there exist proposals addressing alignment [6-8], it is still acknowledged as an open issue for top-management [9], as well as for IT executives [10].

Despite this acknowledged importance of aligning strategic initiatives and plans with IS, this linkage suffers from shortcomings of existing approaches. Established business strategy formulations are typically overlooked and business strategy is abstractly linked to IS models when it comes to IS requirements and other Enterprise Models (EM) [11,12]. While Enterprise Architecture (EA) 
proposals such as TOGAF [13] and the Zachman Framework [14], as well as Business Architecture proposals [15] include business elements or layers that affect IS, they lack on linking them to business strategy formulations $[11,12]$.

These shortcomings are indicative of the ambiguity of business strategy for this alignment linkage, which makes even more difficult to grasp strategic initiatives and facilitate the development of IT solutions. Our model-driven proposal for alignment is based on the Unified Business Strategy Meta-model (UBSMM), which is an integration of the conceptualizations of established business strategy formulations from Strategic Management [11,12]. More specifically, UBSMM integrates business strategy formulations covering three complementary types of strategy-shaping logic; the resource-based type with Strategy Maps and Balanced Scorecards [3], the competition type with the Value Chain [2], the Value Shop and the Value Network [16], and the innovation type with Blue Ocean Strategy [17], which altogether constitute adequate coverage of strategic notions $[11,12]$. Leveraging properties of meta-modeling and model-driven development, UBSMM links business strategy with IS models used for IS requirements, with EM and with EA [11,12].

The objectives of this paper are: to empirically validate that the linkage between business strategy and IS is an issue of concern, and collect insights from the industry about the appropriateness of our model-driven approach, and highlight the benefits offered by UBSMM with respect to the linkage. For the the first, an empirical study is conducted, and for the second, an illustrative case using strategic notions from UBSMM is presented.

The section following includes related work motivating the need for our study. Thereafter, the background and design of the study is presented in section three; section four presents the results of the study and section five discusses our findings. The paper concludes with section six on future research steps.

\section{Related Work}

Despite the acknowledged importance of business-IT alignment, during the past decade a strong empirical motivation for business-IT alignment has been put forward focusing primarily on: (i) the relation between alignment and business performance or (ii) the relation between types of business strategies (such as conservative or innovative) and (iii) the degree of alignment [18].

However, minimal empirical basis seems to exist specifically for the linkage between business strategy and IS. Such empirical work would investigate the need for, and the existence of a formal business strategy formulation and its potential for the use of models for reinforcing alignment. A common type of empirical work found in the business-IT alignment literature is Luftmans highly cited work, with the annually published CIO survey results [10]. However, the focus is mostly on alignment maturity. Moreover, the linkage between business strategy and IS is not only relevant to CIOs but also to all those affecting it, or being affected both from the Business or IT. 
The works of $[19,20]$ also follow a modeling approach, however without employing established business strategy formulations. On the other hand, the work of [21] shares our motivation of natural language-based ambiguity of business strategy formulations, and they strive for a conceptualization of Balanced Scorecards (BSC), which aims at reducing practice variability due to interpretations of publications and reports. The purpose of this conceptualization is to become becoming a basis for building tools that are capable of capturing, analyzing and explaining strategic initiatives and intend.

Another effort also aimed at decreasing the ambiguity of business strategy is the Strategy Markup Language (StratML) solely for document management i.e. focused on providing XML-based specifications for strategic and performance plans and reports [22]. This initiative is not concerned directly with business strategy formulations.

\section{Empirical Study Design}

Designing, conducting and reporting an empirical study constitutes a complex operation involving several steps. For our study, we have adopted Oppenheim's 14-stage framework [23].

Stages one through four refer to going through literature, reflecting upon it, and choosing an appropriate form for the study. Our study aims at empirically validating the relevance of addressing the linkage between business strategy and IS in today's enterprise terrain and collecting insights about using models as an appropriate way of addressing it. Due to the need for reach to practitioners, the design of study selected has been a self-administered online questionnaire. The assumption to be investigated (stage five) is expressed through the three questions formulated in the Introduction.

For the design (stage six) guidelines include establishing objectives, measures and scales, as well as types of questions, layout, wording, flow of questions, and validity concerns. The following objectives have been set for the questionnaire:

Obj. 1: Identify to whom and why is the linkage between business strategy

and IS a concern for the Business and IT actors within a company.

Obj. 2: Identify gaps between strategy and systems development hindering the alignment linkage.

Obj. 3: Confirm the use of models as a relevant solution to address such gaps.

The questionnaire has been built with the free online tool Survey Gizmo (http://www.surveygizmo.com), and consists of 29 questions spreading across six sections. Sections have been derived from the aforementioned objectives; section one focuses on the first objective by capturing whether the linkage between business strategy and IS is a concern and whether this concern is being currently addressed, how, etc. Sections two and three focus on the second objective by capturing respondents' familiarity with strategic formulations, how they are used in their company, and they are used in relation to information systems requirements. Sections four and five focus on the third objective by capturing 
respondents' familiarity with models and how they are used in their company, as well as their views on the utility of a model-driven proposal for alignment. Section six captures demographic information.

All sections include explanations and examples of core concepts and terms used (i.e. information system, requirements, strategy, model), together with information motivating some questions. Types of questions used, include openended, multiple choice, checkboxes, and Likert scale questions. Options offered cover possible alternatives relevant to the questions without overlapping, while units and scales have been consistently used, and double-barreled questions have been avoided [23]. Questions have been neutrally formulated to avoid bias, and questions for consistency checking across answers have been used.

A pilot study has been conducted (stage seven) to assess the questionnaire's validity and understandability [24]. This included a group of four academic experts on business-IT alignment for conformance to the hypothesis defined (construct validity), and sufficient domain coverage (content validity). Additionally, a convenience sample of 52 professionals from around the world has also been used to simulate the realistic setting of the study allowing to test the questionnaire by providing input on all functional aspects (language, structure, layout, etc.). Apart from language, structure and layout improvements, the pilot study resulted a refined set of questions, from 41 down to 29 . The questionnaire can be tested at: http://www.surveygizmo.com/s3/1305947/Strategy-IT-Alignment and questions are available at: $h t t p: / / g o o . g l / 8 r f g f o$.

The sample has been designed (stage eight) following quota sampling, where mutually exclusive sub-groups have been identified. The selection of companies has been stratified across medium and large profit-driven companies registered in Kista, Sweden, based on information provided by the Swedish Agencies Registration Office (http://www.bolagsverket.se). Medium and large companies (more than 50 but less than 250, and more than 251 respectively) have been selected because small and micro companies (less than 50) are typically considered agile and due to size the issue of alignment is not relevant. Also, profit-driven companies have been selected over charity organizations and state-owned companies because the vast majority of business strategy formulations has been defined based on profit-driven companies. Invitation for participation (stage nine) took place via email, stating the objectives of the study, the form and the ethical considerations of handling the data.

The data collection process lasted four weeks (stage eleven) during the late spring and early summer of 2013. Processing the data and statistically analyzing them has been done with the assistance of the aforementioned survey tool (stages twelve and thirteen). This paper constitutes reporting on the questionnaire results and testing of the hypothesis (stage fourteen).

\section{Empirical study Results}

The results of the study include the responses of 45 participants coming from seven large and medium profit-driven companies: two active in manufacturing 
( 8 and 6 participants), one active in software development (5 participants), one active in media and publishing (3 participants), and three active in telecommunications $(7,11$, and 5 participants), which includes a global leader in networking and a Nordic leader in mobile and internet services. All the objectives of the questionnaire have been met in that the results provide answers along with insights from respondents. Results are presented for each of the objectives accompanied with discussions.

\subsection{Objective 1}

Results come from the first section of the questionnaire are summarized in Table 1. They indicate that the linkage between business strategy and IS is an issue of concern for the vast majority of respondents. Apart from the overall results, it is interesting to examine responses from different perspectives due to function served (Business, IT, Both), and size of the company (Medium, Large).

While those who have one distinct function share $100 \%$ the view that the linkage between business strategy and IS is an issue of concern, those serving both functions do no share the same absolute. This could be due to the fact that those serving both functions are expected to have a better understanding of both the Business and IT and thus do not consider the linkage between business and IS to be an issue of concern.

Table 1. Results answering: "Is the linkage between business strategy and IS an issue of concern for your company?".

\begin{tabular}{|l|c|c|}
\hline Population & Yes & No \\
\hline All & $92.5 \%$ & $7.5 \%$ \\
\hline Business & $100 \%$ & - \\
\hline IT & $100 \%$ & - \\
\hline Both & $89 \%$ & $11 \%$ \\
\hline Medium & $90 \%$ & $10 \%$ \\
\hline Large & $100 \%$ & - \\
\hline
\end{tabular}

Table 2. Responses on model types used.

\begin{tabular}{|l|c|}
\hline Model types used & Percentage \\
\hline Business Models & $69.2 \%$ \\
\hline Requirements Models & $61.5 \%$ \\
\hline Process Models & $61.5 \%$ \\
\hline Use Case Models & $42,3 \%$ \\
\hline Goal Models & $23.1 \%$ \\
\hline Enterprise Models & $15.4 \%$ \\
\hline Conceptual Models & $15.4 \%$ \\
\hline Simulation Models & $15.4 \%$ \\
\hline No Models & $7.7 \%$ \\
\hline I do not know & $3.9 \%$ \\
\hline
\end{tabular}

Another grouping presented in Table 1 focuses on the size of the company and shows that the linkage between business strategy and IS is of concern for all large-sized companies (employing $>250$ people) whereas it is for most mediumsized companies (employing between 50 and 250 people). This is anticipated and coincides with our assumption to focus on medium and large companies in this study. The larger the company, the more cumbersome it becomes to align all functions, especially when they are as pervasive across an organization as IT is.

Motivation for being an issue of concern (i.e. the why?) has been acknowledged by respondents due to strategy not being clear enough and due to lack of understanding how IS can enhance and support strategy.

In the same section, those that have acknowledged this linkage as a concern for their company, have also been asked to indicate methods and techniques practiced to address it; $30 \%$ answered that no method or technique is practiced, $15 \%$ answered that their company does not strive for alignment between business strategy and IS and 55\% answered they do not know. 


\subsection{Objective 2}

Results from sections two and three of the questionnaire focus on business strategy and how it relates to systems development within their company, particularly system requirements.

First of all, participants were asked in terms of strategy awareness within their company (Table 3). Those fully and partially aware were asked to identify all forms of communication used for business strategy dissemination (Table: 4).

Table 3. Strategy awareness levels.

\begin{tabular}{|l|c|}
\hline Awareness & Percent \\
\hline Fully aware & $71 \%$ \\
\hline Partially aware & $14 \%$ \\
\hline Not aware & $6 \%$ \\
\hline No strategy & $9 \%$ \\
\hline
\end{tabular}

Table 4. Ways of disseminating strategy within companies.

\begin{tabular}{|l|c|}
\hline Strategy Dissemination & Percent \\
\hline Verbally & $78 \%$ \\
\hline In text (i.e. reports) & $75 \%$ \\
\hline Graphical (i.e. charts) & $44 \%$ \\
\hline
\end{tabular}

When asked whether business strategy dissemination was timely understood and whether the business intent of the company was clearly expressed; $58 \%$ were positive, $9 \%$ negative, and $33 \%$ neutral.

When asked about alignment between business strategy and IS; whether IS requirements were utilized with respect to business strategy, whether there exists synchronicity and traceability between IS and business changes, as well as whether strategic objectives are utilized in IS development (directly or indirectly); $33 \%$ were positive, $31 \%$ negative, and $36 \%$ neutral.

Overall, $59 \%$ of respondents claim strategy is aligned with IT in their companies, and $41 \%$ claim it is not. Those who responded no identified reasons hindering the linkage between business strategy and IS in their companies:

For $\mathbf{7 3 \%}$ not enough communication exists between The Business and IT,

For $37 \%$ strategy is not communicated at all,

For $37 \%$ IS is not related to strategy,

For $\mathbf{2 7 \%}$ strategy is not understood at all,

For 27\% strategy is not expressing the company's real strategic intent.

A significant conclusive observation from this section is that $85 \%$ answered they are fully and partially aware of their company's strategy suggesting that business strategy is disseminated. However, $41 \%$ claim strategy is not aligned with IS and the most significant reason for this gap seems to be the fact that there exists not enough communication between the Business and IT (73\%).

\section{$4.3 \quad$ Objective 3}

Results come from sections four and five of the questionnaire, which focus on models and their utilization for alignment within respondents' companies.

Table 2 presents the types of models most widely used, as indicated by respondents: business, requirements, process, and use case are dominating.

While $81 \%$ of respondents have indicated they are familiar with models, for Objective 3 we have selected only those that have indicated a certain level of competency regarding models. The reason behind this selection is that a certain 
level of knowledge and familiarity with models is needed to assess the use of models for model-driven alignment. The scale of familiarity included novice, experienced beginner, practitioner, knowledgeable practitioner and expert. We have selected the answers from those that have indicated they are practitioners on at least one of the model types shown in table 2 . This filtering resulted into 19 respondents and results from their answers are presented along with results from answers coming from the total sample (Table 5).

Table 5. Positive responses on the use of Table 6. Correlation between familiarity models.

\begin{tabular}{|l|c|c|}
\hline Statements & IT & Total \\
\hline $\begin{array}{l}\text { Our company has the know-how } \\
\text { to use models for model-driven } \\
\text { alignment }\end{array}$ & $32 \%$ & \\
\hline $\begin{array}{l}\text { Modeling our strategy would im- } \\
\text { prove alignment of strategies } \\
\text { across units }\end{array}$ & $72 \%$ & $68 \%$ \\
\hline $\begin{array}{l}\text { Modeling our strategy would im- } \\
\text { prove alignment towards partners }\end{array}$ & $56 \%$ & $52 \%$ \\
\hline $\begin{array}{l}\text { Modeling our strategy would bring } \\
\text { value to our company }\end{array}$ & $72 \%$ & $50 \%$ \\
\hline $\begin{array}{l}\text { Modeling our strategy could im- } \\
\text { prove the linkage between strategy } \\
\text { and IS for our company }\end{array}$ & $89 \%$ & $84 \%$ \\
\hline
\end{tabular}
with models and the use of models improving the alignment linkage.

\begin{tabular}{|l|c|c|c|}
\hline $\begin{array}{l}\text { Familiarity with } \\
\text { Models }\end{array}$ & $\rho$ & $R^{2}$ & $p<0.05$ \\
\hline Enterprise Models & 0.5474 & $29.96 \%$ & 0.0001 \\
\hline Process Models & 0.6518 & $42.48 \%$ & $<0.00001$ \\
\hline Business Models & 0.7112 & $50.58 \%$ & $<0.00001$ \\
\hline Requirements Models & 0.4157 & $17.28 \%$ & 0.004513 \\
\hline Conceptual Models & 0.3704 & $13.72 \%$ & 0.012259 \\
\hline Information Models & 0.5306 & $28.16 \%$ & 0.000177 \\
\hline Simulation Models & 0.3899 & $15.20 \%$ & 0.000195 \\
\hline Goal Models & 0.5278 & $27.86 \%$ & 0.008106 \\
\hline Use Case Models & 0.5817 & $33.83 \%$ & $2.8 \mathrm{E}-05$ \\
\hline
\end{tabular}

Respondents have also provided motivation over their positive assertion on the improvement of the alignment linkage between business strategy and IS due to the use of models. The motivations mostly refer to benefits that models bring, such as structure, less ambiguity, understandability, automation, experimentation for possible alternatives and hypothesis testing.

Furthermore, results have shown that there exists a moderate positive correlation between respondents' familiarity with models and agreement that the use of models would lead to improvement of alignment between business strategy and IS. Correlation indicates the strength of the statistical relationship between questions (using Likert scales in this case) but cannot determine cause and effect as in which one is influencing the other.

Scatterplots of two such moderate positive correlations are presented in figure 1 for familiarity with Enterprise models (left) and Business models (right). The vertical axes is scaled 0-5 capturing familiarity with models: 0 for not familiar at all, 1 for novice, 2 for experienced beginner, 3 for practitioner, 4 for knowledgeable practitioner, and 5 for expert. The horizontal axes is scaled 1-5 capturing confidence for improvement of alignment through the use of such models: 1 for strongly disagree, 2 somewhat disagree, 3 I do not know, 4 somewhat agree, 5 strongly agree. This means there is a tendency among those with high familiarity with enterprise models and business models (practitioners and experts) to express higher levels of agreement with the use of models improving alignment.

Table 6 presents the correlation coefficient $\rho$ (second column), the coefficient determination $\mathbf{R}^{2}$ (third column) and the statistical significance $\mathbf{p}$ (fourth column) between familiarity with models and agreement with the use of models improving alignment. For enterprise models, business models, process models, 
information models, goal models and use case models the correlation is moderate positive as $\rho$ is around +0.5 and moderate percentages of data close to the regression line (27\%-51\%). For requirements models, conceptual models and simulation models the correlation is weak positive as $\rho$ is closer to 0 and low percentages of data close to the regression line (13\%-18\%). For all correlations reported there is a $5 \%$ likelihood they are a result of chance due to the probability threshold is set: $\mathbf{p}<0.05$.
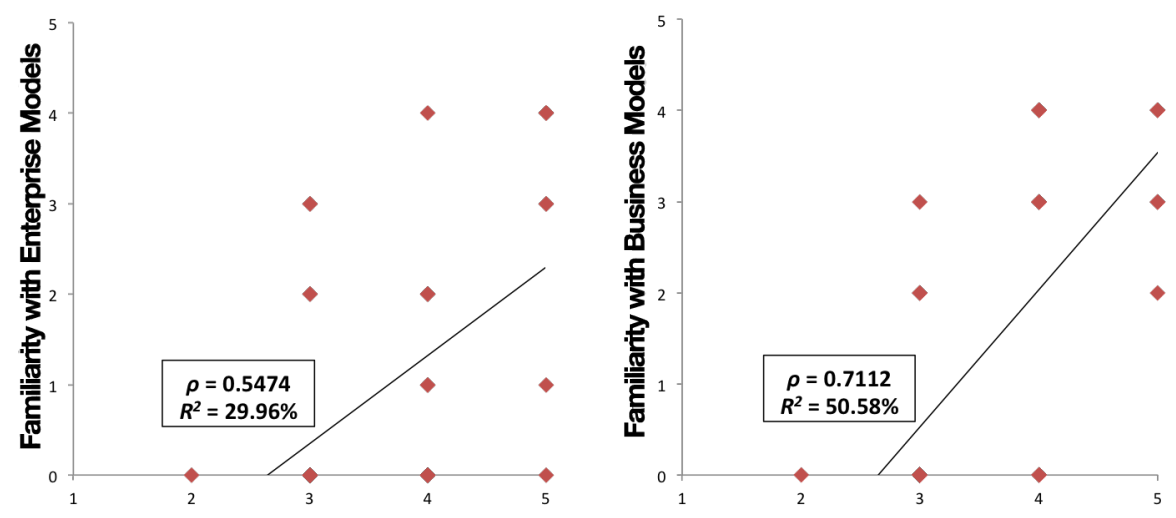

Using models would lead to improvements of alignment between business strategy and IS

Fig. 1. Familiarity with Enterprise models (left) and Business models (right).

\section{Discussion}

Business strategy formulations are typically natural language-based, usually accompanied by schematic representations. The ambiguity of such formulations risks their dissemination to be subject of interpretation. The study's results have indicated the most significant hindering factor is insufficient communication between the Business and IT (objective 2 of the study). This difficulty can be overcome by conceptualizing notions of business strategy formulations and thereafter mapping them onto notions of techniques and methods used for IS requirements. Clear semantics are set for strategy notions which facilitate unambiguous understanding and dissemination of business strategy to IS. This does not leave space for interpretation and makes changes less prone to creating problems. Traceability makes the impact of changes in strategy traceable to IS (i.e. requirements, features, etc.)

The use of models has been acknowledged by respondents as a solution to improve the linkage between business strategy and IS (objective 3 of the study). Results have allowed the identification of correlations between familiarity with models and anticipation that the use of models for the linkage between business strategy and IS improves alignment. Particular types of models showed stronger correlations than others (e.g. business and process) suggesting that proposals like ours should focus on these model types for mapping business strategy to IS. 
In a broader scope, conceptualizing business strategy promotes meaningful transparency of strategic initiatives across an organization, by making such information available. This enhances internal coordination within an organization as it establishes a shared vocabulary about customers, products, processes and activities, which creates a common base for understanding addressing the problem of business strategy and strategic initiatives being open to interpretation. Decreased ambiguity leads to improvements in automation of mappings towards IT solutions, and thus can ideally increase efficiency of business strategy implementation. Business strategy modeling can be the basis for building patterns when linking different strategy initiatives to IT solutions, which increases organizational agility to shift from one initiative to another.

Finally, with respect to the aforementioned benefits of business strategy modeling and the use of UBSMM, certain limitations should also be mentioned. An obvious limitation is the lack of the techniques and the tools to support development of UBSMM to facilitate mappings towards IS (i.e. process models, enterprise models, requirements models, etc. as well as EM and EA as it is the case with system development tools. Another limitation concerns the scope and extent of evaluation for UBSMM due to the fact that a full scale case study requires a long period of handling business sensitive information, thus being limited to small real-world case studies, published cases and reports [11] such as the one of Southwest Airlines.

\section{Concluding Remarks \& Future Work}

In this paper, we have argued for the need to enrich the current body of knowledge on the alignment linkage by empirically validating that the linkage between business strategy and IS is still an issue of concern. In that line, we have conducted a social study in the form of an online self-administered questionnaire. We have presented the design steps taken and we have also reported on the results of the study by addressing each one of the three objectives set for the questionnaire.

The objectives of this paper has been met in that our findings, within their limitations, constitute a current empirical contribution that justifies the theoretical basis of our proposal for model-driven alignment. Results have validated that linkage between business strategy and IS remains an open issue of concern and addressing it methodically is still suffering. Moreover, results have also indicated business strategy is not utilized in IS development with insufficient communication being the major hindering factor. Finally, results regarding the use of models for the linkage between business strategy and IS are positively received by respondents.

Based on the findings of our study, succeeding steps of our future research have two main directions. One path includes conducting further empirical studies to gain more insights and examine larger populations. Another line of work is driven towards conducting case studies using UBSMM to further assess the utility and applicability of our proposal for model-driven alignment. 


\section{References}

1. Burns, T., Stalker, G.M.: The Management of Innovation. University of Illinois at Urbana-Champaign's Academy for Entrepreneurial Leadership Historical Research Reference in Entrepreneurship. Tavistock, London, UK (1961)

2. Porter, M.E.: Competitive advantage: Creating and sustaining superior performance. Free Press (1985)

3. Kaplan, R., Norton, D.: Strategy maps: Converting intangible assets into tangible outcomes. Harvard Business Press (2004)

4. Leede, J.d., Looise, J.C., Alders, B.C.: Innovation, improvement and operations: an exploration of the management of alignment. International Journal of Technology Management 23(4) (2002) 353-368

5. Ross, J.W., Weill, P., Robertson, D.C.: Enterprise architecture as strategy: Creating a foundation for business execution. Harvard Business Press (2006)

6. Henderson, J.C., Venkatraman, N.: Strategic alignment: a framework for strategic information technology management. (1989)

7. Luftman, J.N., Bullen, C.V., Liao, D., Nash, E., Neumann, C.: Managing the information technology resource: Leadership in the information age. Pearson Education Upper Saddle River (2004)

8. Chan, Y.E., Reich, B.H.: It alignment: what have we learned? Journal of Information technology 22(4) (2007) 297-315

9. Luftman, J., Kempaiah, R., Nash, E.: Key issues for it executives 2004. MIS Quarterly Executive 4(2) (2005) 269-285

10. Luftman, J., Derksen, B.: Key issues for it executives 2012: Doing more with less. MIS Quarterly Executive 11(4) (2012) 207-218

11. Giannoulis, C.: Model-driven Alignment: Linking Business Strategy with Information Systems. PhD thesis, Stockholm University (May 2014)

12. Giannoulis, C., Zdravkovic, J., Petit, M.: Model-driven strategic awareness: From a unified business strategy meta-model (ubsmm) to enterprise architecture. In: Enterprise, Business-Process and Information Systems Modeling. Springer (2012) 255-269

13. Forum: The open group architectural framework (togaf) version 9.1. Technical report, The Open Group (2013)

14. Zachman, J.A.: A framework for information systems architecture. IBM systems journal 26(3) (1987) 276-292

15. Versteeg, G., Bouwman, H.: Business architecture: A new paradigm to relate business strategy to ict. Information Systems Frontiers 8(2) (2006) 91-102

16. Stabell, C.B., Fjeldstad, Ø.D.: Configuring value for competitive advantage: on chains, shops, and networks. Strategic management journal 19(5) (1998) 413-437

17. Kim, W.C., Mauborgne, R.: Blue Ocean Strategy. Harvard Business Review Press (2004)

18. Chan, Y.E., Sabherwal, R., Thatcher, J.B.: Antecedents and outcomes of strategic is alignment: an empirical investigation. Engineering Management, IEEE Transactions 53(1) (2006) 27-47

19. Champion, R., Moores, T.T.: Exploiting an enterprise model during systems' requirements capture and analysis. In: Requirements Engineering, 1996., Proceedings of the Second International Conference on, IEEE (1996) 208-215

20. Opdahl, A.L.: Model-supported alignment of information systems architecture. Business strategies for information technology management (2003) 28 
21. Group, B.A.W.: Balanced scorecard metamodel. Technical report, The Object Mangement Group (OMG) (2010)

22. ANSI/AIIM: Standard recommended practice - strategy markup language - part 1: Stratml core. Technical Report 21:2009, American National Standards Institute (ANSI) (2009)

23. Oppenheim, A.N.: Questionnaire design, interviewing and attitude measurement. Continuum International Publishing Group (2000)

24. Giannoulis, C., Zdravkovic, J., Petit, M.: Model-centric strategy-it alignment: An empirical study in progress. In: Advanced Information Systems Engineering Workshops, Springer (2013) 146-155 